\title{
The Effect of Fibroblast Growth Factor 2 (FGF2) on Mesenchymal Stromal Cell Differentiation
}

\author{
Kähkönen Tiina, Ivaska Kaisa K, Härkönen Pirkko
}

University of Turku , Institute of Biomedicine, Department of Cell Biology and Anatomy, Turku, FINLAND

\section{INTRODUCTION}

Mesenchymal stromal cells (MSC) have a potential to differentiate to osteoblasts and adipocytes. Differentiation can be stimulated or inhibited by different growth factors, including fibroblast growth factors (FGFs). FGF2 is produced by osteoblasts and bound to bone matrix upon mineralization. It is also expressed in white adipose tissue. However, the studies on the effects of FGF2 on MSC differentiation have provided controversial results. The aim of this study was to evaluate the effects of short- and long-term FGF2 treatment on MSC differentiation to osteoblasts and adipocytes.

\section{METHODS}

Mouse MSC-derived cells were cultured in the medium that led to differentiation of MSCs to osteoblasts in 14 days and to adipocytes in 7 days. The cells were treated during the differentiation with control (DMSO), FGF2 $(25 \mathrm{ng} / \mathrm{ml})$, the FGFR inhibitor PD173074 (100nM) or combination of the two for $24 \mathrm{~h}$ prior to sample collection (short-term treatment) or through the whole culture period (long-term treatment). RNA samples were collected for qRT-PCR analysis of osteblast or adipocyte marker gene and FGF-receptor (FGFR) expression.

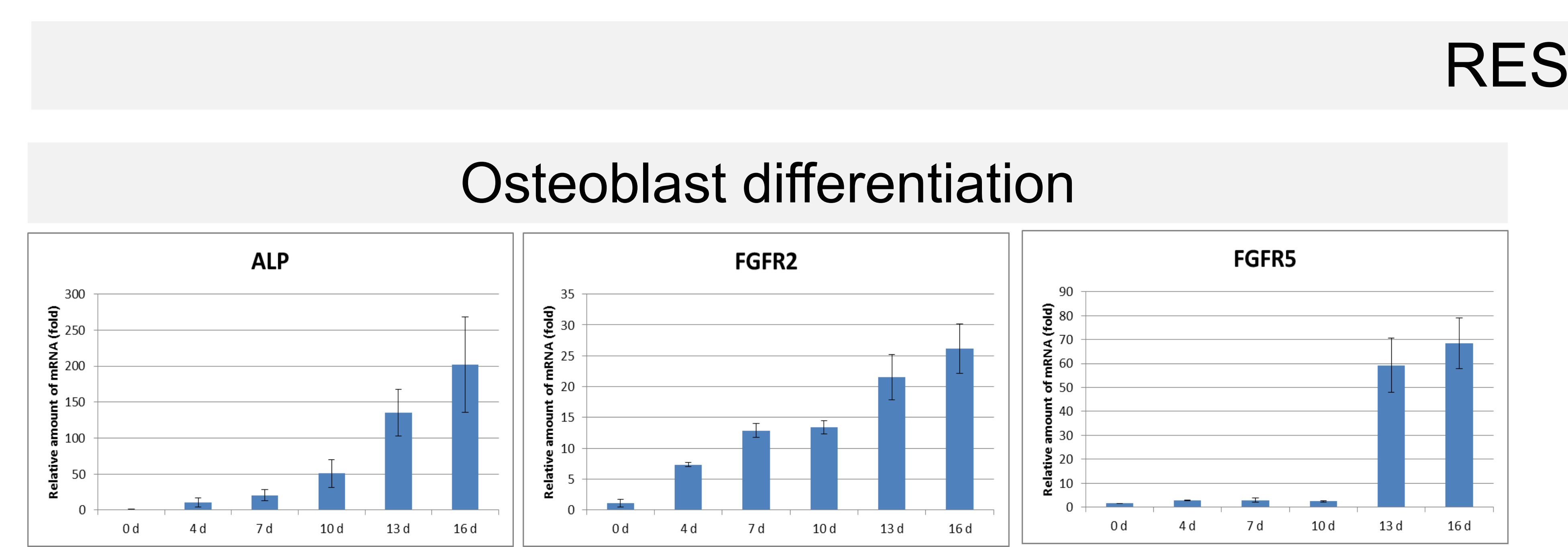

During osteoblast differentiation the expression of osteoblast marker genes, COL1, OC, ALP and RUNX2, increased. Simultaneously the mRNA levels of FGFRs increased, remarkably those of FGFR2 and FGFR5.

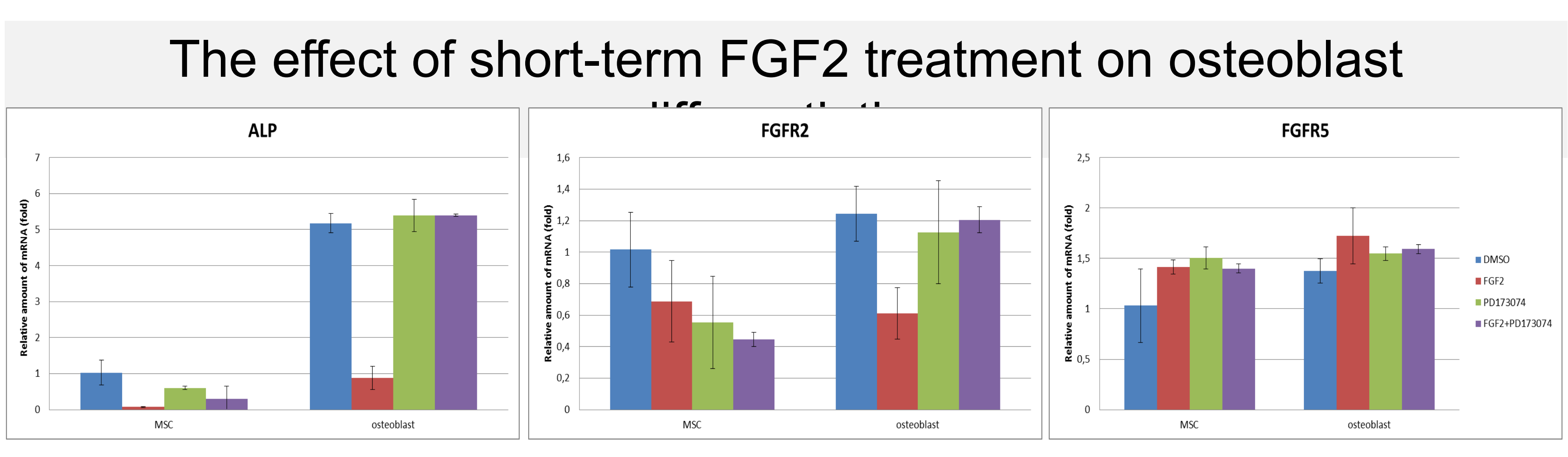

Short-term FGF2 treatment decreased the expression of COL1, OC, ALP and RUNX2 mRNA levels. The mRNA levels of FGFR2 were decreased due to FGF2 treatment whereas FGF2 had no effect on the mRNA expression of FGFR5.

The effect of long-term FGF2 treatment on osteoblast differentiation

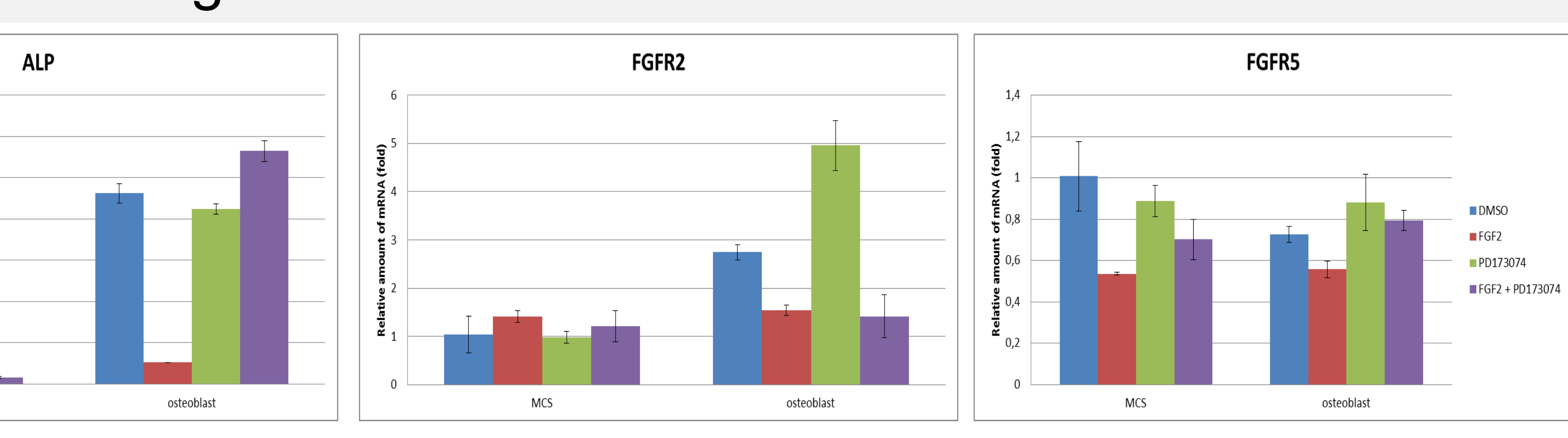

Long-term FGF2 treatment decreased mRNA levels of osteoblast marker genes. Interestingly the mRNA levels of ALP and RUNX2 were slighly increased by a combination treatment of FGF2 and PD173074. A long-term FGF2 treatment decreased the expression of FGFR2 and FGFR5 mRNA whereas a long-term treatment with PD173074 increased the mRNA expression of FGFR2.

\section{RESULTS}

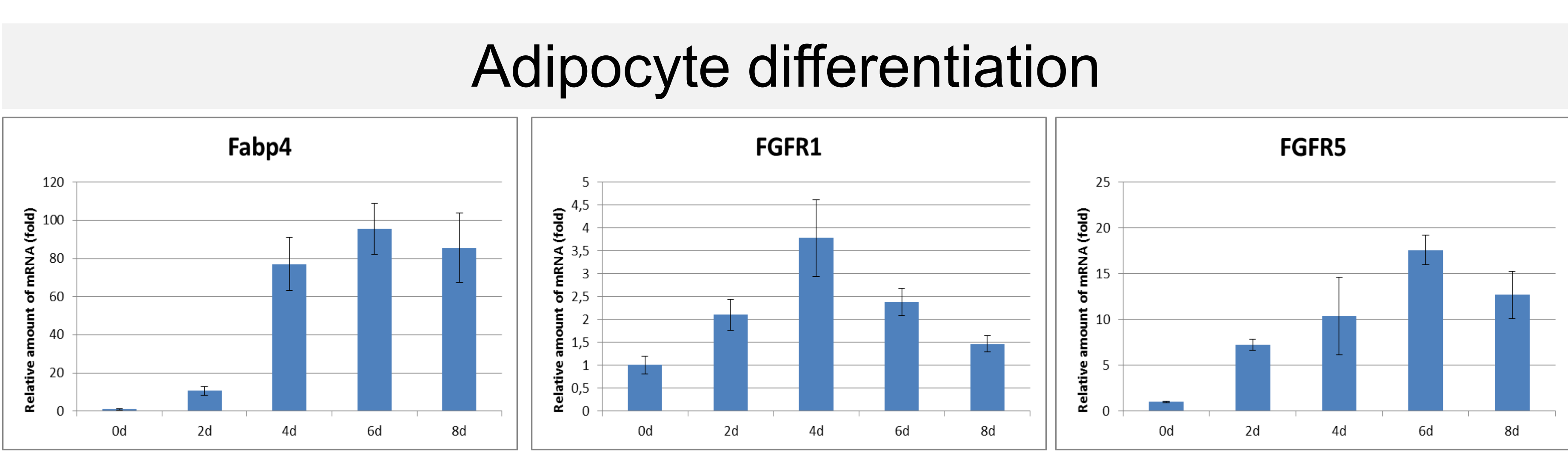

During adipocyte differentiation expression of mRNA levels of the adipocyte marker genes FABP4 and PPARy increased.

Simultaneously, we observed an increase in FGFR1 and FGFR5 mRNA levels.

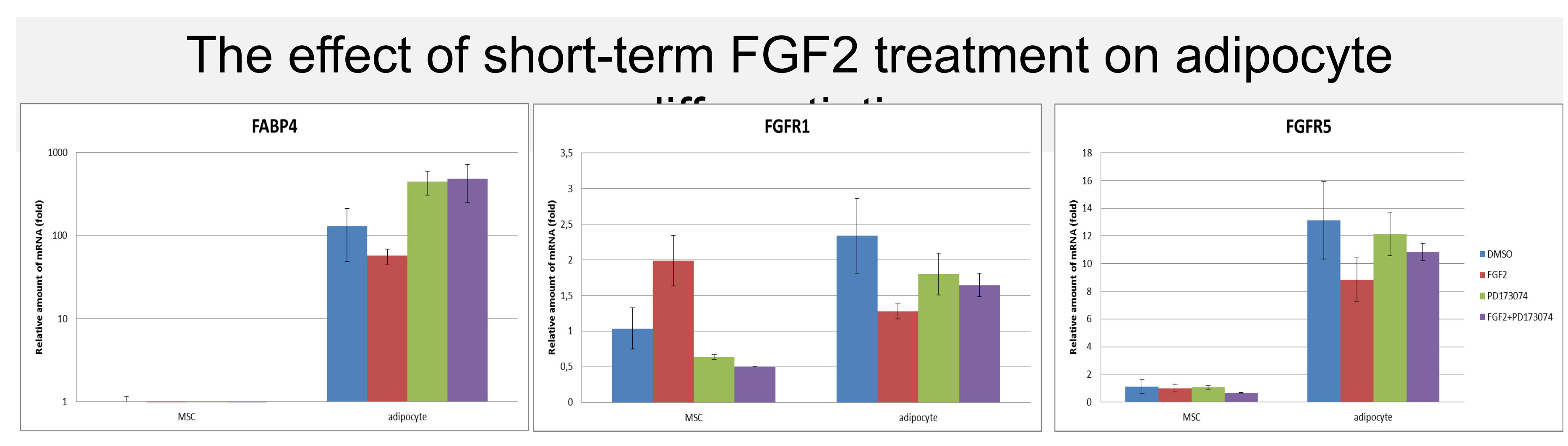

Short-term FGF2 treatment decreased expression of the adipocyte marker genes PPARy and FABP4. The mRNA levels of FGFR1 and FGFR5 were decreased in adipocytes treated with FGF2.

The effect of long-term FGF2 treatment on adipocyte differentiation

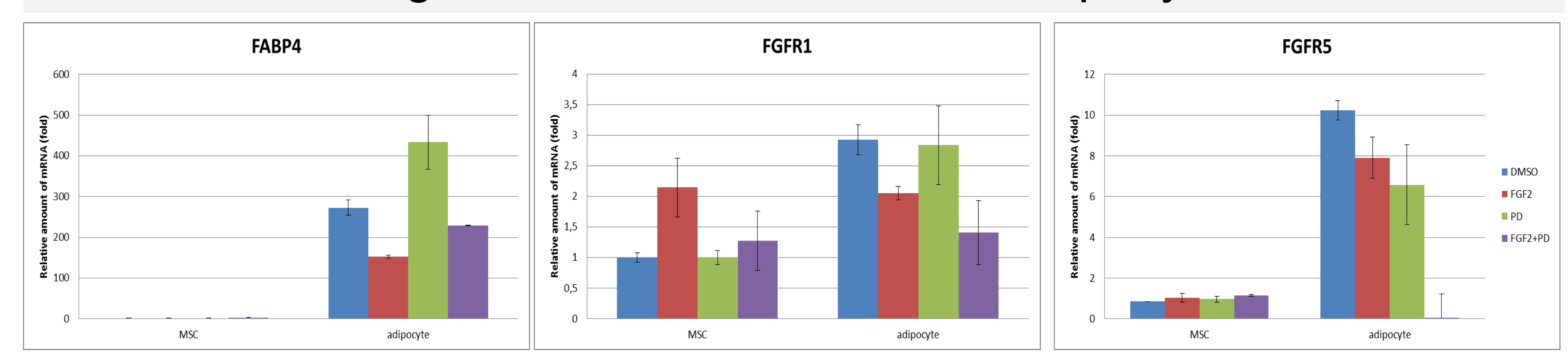

Long-term FGF2 treatment decreased the mRNA expression of FABP4 and slighly the mRNA expression of PPARy. A long-term treatment with PD173074 increased the expression of adipocyte marker genes. The mRNA levels of FGFR1 and FGFR5 were decreased by a long-term FGF2 treatment.

\section{CONCLUSIONS}

- Short- and long-term treatments with FGF2 inhibit the differentiation of MSCs to both osteoblast and adipocyte lineages indicated by a decrease in marker gene expression

- During osteoblast differentiation a short-term treatment of MSC with FGF2 downregulated the expression of FGFR2 mRNA. A long-term treatment decreased the expression of FGFR2 and FGFR5 mRNA

- During adipocyte differentiation both a short-term and long-term treatment with FGF2 downregulated the expression of FGFR1 and FGFR5 mRNA 